\title{
Clinical and Radiological Diagnosis of Rarely Seen OSMED Syndrome
}

\author{
Gulay Gulec Ceylana, d, Tulay Tos ${ }^{\mathrm{b}}$, Ahmet Resat Dogusan ${ }^{\mathrm{c}}$
}

\begin{abstract}
Otospondylomegaepiphyseal dysplasia (OSMED) (MIM 215150) is an autosomal recessive syndrome. It is a skeletal dysplasia characterized by multiple skeletal anomalies, flat nasal bridge, mid-face hypoplasia, anteverted nostrils and sensorineural hearing loss. In this case report, we evaluated a 3-year-old Turkish girl born to consanguineous parents. She had a medical history of bilateral sensorineural hearing loss, frontal bossing and strabismus. The radiographic findings supported OSMED syndrome with her phenotype. Our aim was to facilitate the early diagnosis of this rarely seen syndrome and to contribute to the natural history of patients with OSMED syndrome.
\end{abstract}

Keywords: OSMED; Short stature; Epiphyseal dysplasia; Deafness

\section{Introduction}

Otospondylomegaepiphyseal dysplasia (OSMED) (MIM 215150) is an autosomal recessive skeletal dysplasia [1]. It is characterized by sensorineural hearing loss, enlarged epiphyses, disproportionately short limbs, vertebral body anomalies and characteristic facies. The facial dysmorphism is characterized by mid-face hypoplasia and a flat nasal bridge, small upturned nostrils, a long philtrum, cleft palate/bifid uvula, micrognathia, and hypertelorism. During the second decade of life, joint pain and restricted mobility of the finger joints can appear $[2,3]$. The radiographic findings include short dumbbell-shaped long bones, large epiphyses, and mild platyspon-

Manuscript accepted for publication February 11, 2016

aDepartment of Medical Genetics, Medical School, Yildirim Beyazit University, Ankara, Turkey

bDepartment of Medical Genetics, Sami Ulus Pediatrics Education and Training Hospital, Ankara, Turkey

'Department of Pediatrics, Sami Ulus Pediatrics Education and Training Hospital, Ankara, Turkey

${ }^{\mathrm{d}}$ Corresponding Author: Gulay Gulec Ceylan, Department of Medical Genetics, Medical School, Yildirim Beyazit University, Ankara, Turkey.

Email: gulayceylan23@gmail.com

doi: http://dx.doi.org/10.14740/jmc2431w dyly with coronal clefts. It is a very rarely seen syndrome. It is usually caused by bi-allelic mutations in the "collagen, type XI, alpha 2 gene" (COL11A2) (6p21.3) [4].

In this report, we present a Turkish girl with short limbs, vertebral body abnormalities and a characteristic face appearance. Our diagnosis is based on the clinical and radiographic findings.

\section{Case Report}

A 3-year-old girl was admitted to our clinic with a medical history of bilateral sensorineural hearing loss, frontal bossing and strabismus. She was born to healthy first-cousin parents. There was gestational diabetes during the prenatal period and the ultrasound examination revealed depressed nasal bridge. Her birth weight was 3,500 g and length was $48 \mathrm{~cm}$. Head circumference was not recorded. There was no similar individual in the family. She had two healthy siblings. At the age of 3 years, her weight was $12 \mathrm{~kg}$ (25th - 50th centile), her height was $84 \mathrm{~cm}$ (25th - 50th centile), and her head circumference was $49 \mathrm{~cm}$ (above 97th centile). There was macrocephaly, a flat face appearance, frontal bossing, synophrys, depressed nasal bridge, antevert nostrils and small nose. We also observed short hands and short fingers (Fig. 1). There was mesomelic shortening of upper and lower extremities. There was macrocephaly and a J-shaped sella turcica on radiograph of the cranium. The radiographic examination of the thoracolumbar spine demonstrated thoracolumbar hump and mild platyspondyly. The radiographic examination of upper and lower extremities showed shortening of humerus, radius, ulna femur, tibia and fibula. Radiograph of the pelvis showed squared iliac wings (Fig. 2). Routine biochemistry tests and blood count were normal. Ophtalmology and cardiology consultations were reported as normal. The cytogenetic analysis of peripheral leukocytes showed a normal 46, XX female karyotype. The informed consent of the parents was taken.

\section{Discussion}

According to our clinical, radiographic and laboratory findings, the most likely diagnosis in our patient was OSMED syndrome. Only a few patients with OSMED syndrome have been 


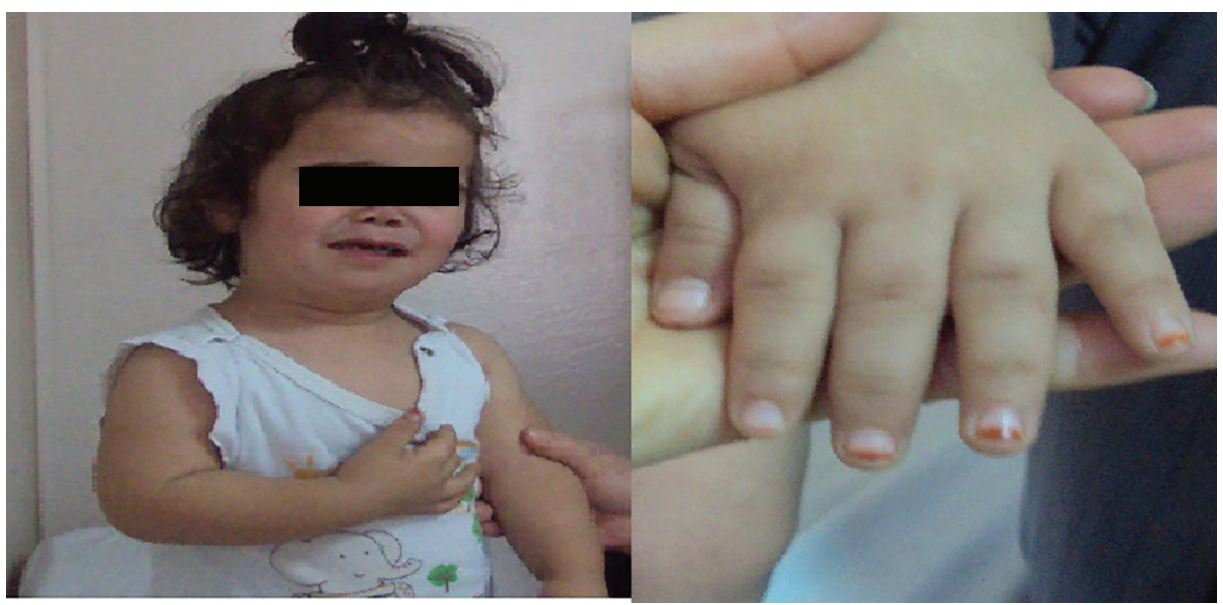

Figure 1. The view of the patient from the front and the view of the patient's hands.

reported $[5,6]$. Our patient showed the major findings of this syndrome such as bilateral sensorineural hearing loss, midface hypoplasia with a flat nasal bridge, anteverted nostrils, and mesomelic shortening of upper and lower extremities. The radiographic changes also supported OSMED syndrome with the findings of shortening of humerus, radius, ulna femur, tibia, fibula and squared iliac wings. Short hands and fingers were other common findings of this syndrome that are also present in this patient [1].

The phenotype of OSMED syndrome resembles that of a group of skeletal disorders. These are Stickler syndrome type 3, Weisenbacher-Zweymuller syndrome (WZS), Marshall syndrome and Kniest dysplasia [2]. In Marshall syndrome and Kniest dysplasia, there are ocular symptomes like myopia, congenital cataracts, glaucoma, etc. [7]. In our patient, no ocular anomalies were found. The other symptomes in our case are similar to Stickler syndrome type 3 and WZS, but these disorders show autosomal dominant inheritance, and only OSMED

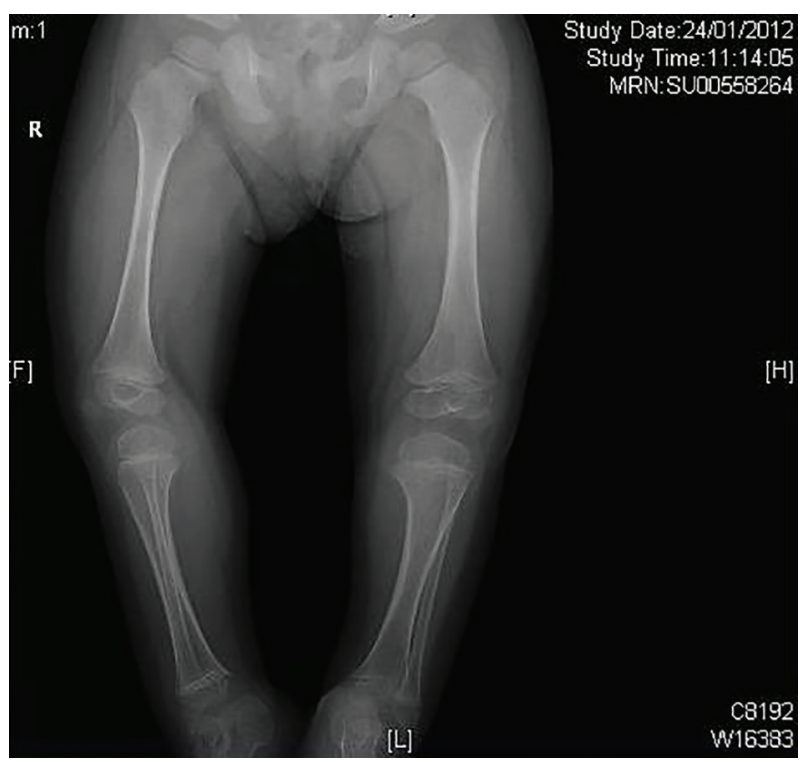

Figure 2. The radiographic view of lower extremities. is inherited as an autosomal recessive trait. These findings confirm our diagnosis [2].

OSMED syndrome is a very rarely seen syndrome. To diagnose the syndrome as early as possible will be beneficial for normal development of the patients. Genetic counselling may be an important step for the affected patients and their families.

\section{Conclusion}

In this case report, we aimed to help clinicians for the diagnosis of a rare syndrome in the early stages. Although there is no spesific treatment for OSMED syndrome, the early diagnosis can be important at the symptomatic treatment of the patients.

\section{Conflict of Interest}

The authors declare that that there is no conflict of interest.

\section{References}

1. Melkoniemi M, Brunner HG, Manouvrier S, Hennekam R, Superti-Furga A, Kaariainen H, Pauli RM, et al. Autosomal recessive disorder otospondylomegaepiphyseal dysplasia is associated with loss-of-function mutations in the COL11A2 gene. Am J Hum Genet. 2000;66(2):368377.

2. Karaer K, Rosti RO, Torun D, Sanal HT, Bahce M, Guran S. A case with oto-spondylo-mega-epiphyseal-dysplasia (OSMED): the clinical recognition and differential diagnosis. Turk J Pediatr. 2011;53(3):346-351.

3. Kayserili H, Wollnik B, Guven G, Emiroglu MU, Baserer $\mathrm{N}$, Uyguner ZO. A novel homozygous COL11A2 deletion causes a C-terminal protein truncation with incomplete mRNA decay in a Turkish patient. Am J Med Genet A. 2011;155A(1):180-185.

4. Harel T, Rabinowitz R, Hendler N, Galil A, Flusser H, 
Chemke J, Gradstein L, et al. COL11A2 mutation associated with autosomal recessive Weissenbacher-Zweymuller syndrome: molecular and clinical overlap with otospondylomegaepiphyseal dysplasia (OSMED). Am J Med Genet A. 2005;132A(1):33-35.

5. van Steensel MA, Buma P, de Waal Malefijt MC, van den Hoogen FH, Brunner HG. Oto- spondylo-megaepiphyseal dysplasia (OSMED): clinical description of three patients homozygous for a missense mutation in the COL11A2 gene. Am J Med Genet. 1997;70(3):315-323.
6. Vikkula M, Mariman EC, Lui VC, Zhidkova NI, Tiller GE, Goldring MB, van Beersum SE, et al. Autosomal dominant and recessive osteochondrodysplasias associated with the COL11A2 locus. Cell. 1995;80(3):431-437.

7. Online Mendelian Inheritance in $\operatorname{Man}, \operatorname{OMIM}(\mathrm{TM})$ : McKusick-Nathans Institute for Genetic Medicine, Johns Hopkins University (Baltimore,MD) and National Center for Biotechnology Information, National Library of Medicine (Bethesda, MD). World Wide Web URL: http:// omim.org/. 